\title{
SOX Gene Expression in Human Osteoarthritic Cartilage
}

\author{
Jochen Haag Pia M. Gebhard Thomas Aigner \\ Institute of Pathology, University Hospital Leipzig, Leipzig, Germany
}

\author{
Key Words \\ Cartilage $\cdot$ Osteoarthritis $\cdot$ SOX transcription factor $\cdot$ Gene \\ expression
}

\begin{abstract}
Objective: While the developmental role of the SOX transcription factors in fetal chondrocyte differentiation is well documented, much less is known about the expression of SOX family members in normal and osteoarthritic adult cartilage. Therefore, the aim of the present study was to present a thorough analysis of SOX gene expression in normal and osteoarthritic human adult cartilage. Methods: RNA from normal and osteoarthritic knee cartilage from human adults was analyzed by gene expression profiling using GeneChip technology (Affymetrix) and quantitative real time PCR. $\boldsymbol{R e}$ sults: Most members of the SOX transcription factor family showed no or very low expression levels in normal and osteoarthritic cartilage from adults. In contrast, SOX9 expression was fairly high in normal cartilage, amounting to approximately $20 \%$ of GAPDH levels. SOX9 transcript levels were substantially reduced in osteoarthritis. SOX6 levels were reduced, albeit starting from a low basis expression in normal tissue. Conclusion: The presented data indicate that the role of the SOX transcription factor family in adult human cartilage is most probably restricted to a few members, with SOX9 being the most prominent. Furthermore, the reduction of SOX9 and SOX6 transcript levels in osteoarthritic chondrocytes might be responsible for the loss of phenotypic stability of osteoarthritic chondrocytes.
\end{abstract}

Copyright $\odot 2008$ S. Karger AG, Basel
(C) 2008 S. Karger AG, Basel

$1015-2008 / 08 / 0753-0195 \$ 24.50 / 0$

Fax +4161306 1234

E-Mail karger@karger.ch

www.karger.com
Accessible online at:

www.karger.com/pat

\section{Introduction}

Members of the SOX family of transcription factors play a prominent role in the early steps of chondrocyte differentiation during fetal development [1]. SOX9 is expressed early in mesenchymal condensations and has an essential function in the subsequent differentiation of prechondrogenic precursor cells into chondrocytes $[2,3]$. This is highlighted by the fact that mutations of the SOX 9 gene can cause a syndrome known as campomelic dysplasia, which is characterized by severe skeletal malformations [4]. SOX9 expression is closely correlated with the expression of collagen type II during skeletogenesis and the protein has been shown to bind to chondrocytespecific enhancer elements of the col2al gene [5-8]. SOX5 and SOX6, which belong to another subgroup of SOX proteins, are coexpressed with SOX9 during chondrogenic differentiation, and, in cooperation with SOX9, activate the transcription of collagen type II and aggrecan [9]. While the expression pattern and the role of the SOX5, SOX6 and SOX9 proteins during development is well studied, much less is known about the expression and regulation of these proteins and other SOX family members in normal and osteoarthritic cartilage from adult humans. Therefore, we decided to conduct a thorough survey of SOX transcription factor mRNA expression in adult human cartilage including arthritically degenerated tissue. 
Table 1. Sequences of primers and probes used for quantitative real time PCR experiments

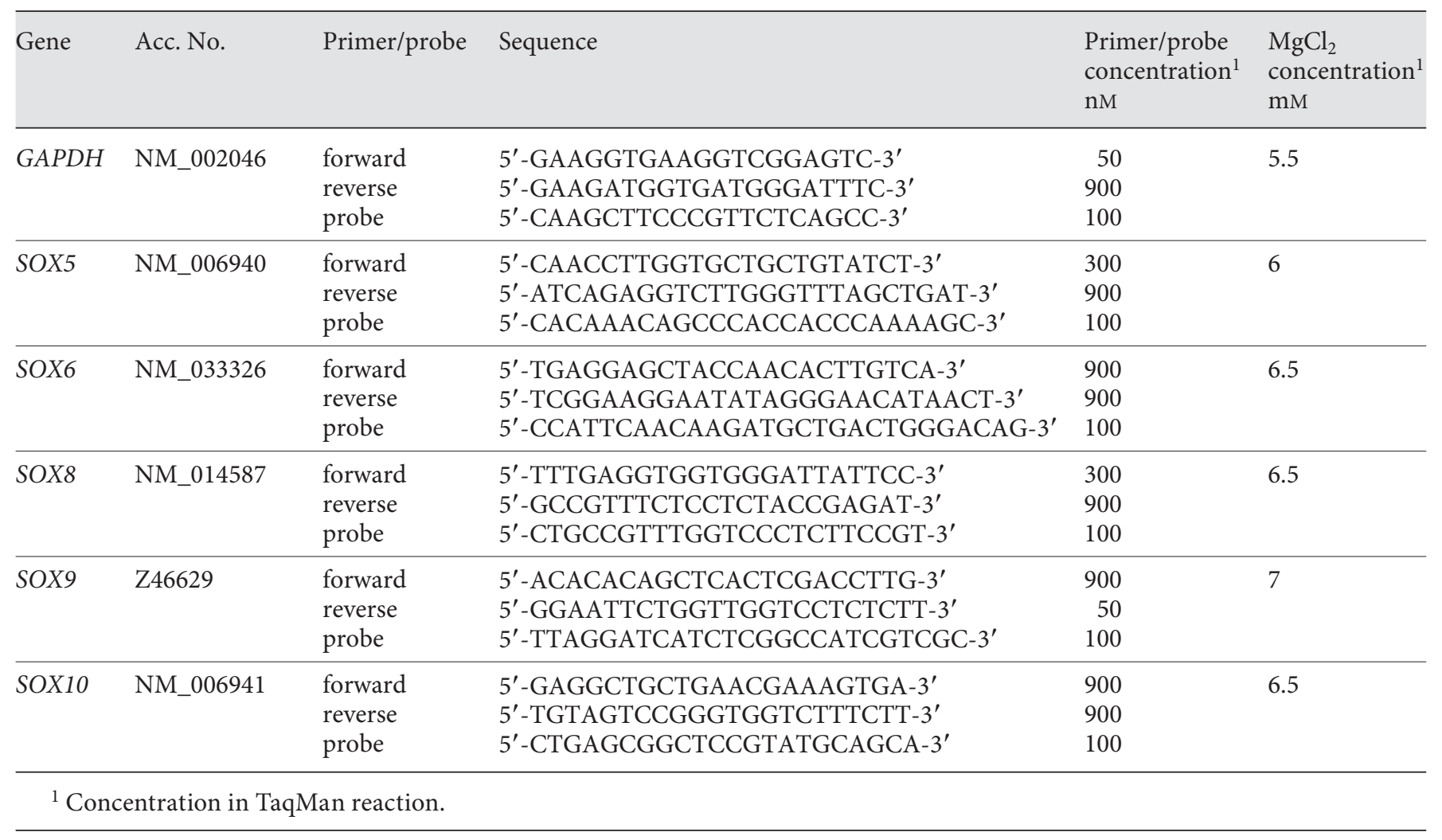

\section{Materials and Methods}

\section{Cartilage Samples}

Cartilage from human femoral condyles was processed as described previously [10]. Normal articular cartilage and cartilage from early degenerative lesions were obtained from autopsies within $48 \mathrm{~h}$ of death. Osteoarthritic cartilage was obtained during total knee replacement surgery. Cartilage was considered to be normal if it showed no significant softening or surface fibrillation. Cases of rheumatoid arthritis were excluded from the study. Only primary degenerated and not regenerative cartilage (osteophytic tissue) was used for RNA isolation. Cartilage samples contained no bone or bone marrow. For quantitative real time PCR studies, 8 samples of normal articular cartilage (subjects: $48-83$ years, mean 64.5 years), 8 samples of cartilage from early degenerative lesions (subjects: $59-86$ years, mean 72.9 years) and 9 samples of osteoarthritic cartilage (subjects: $60-83$ years, mean 70.0 years) were analyzed. For gene expression profiling, cartilage samples were used from 13 normal individuals (subjects: 48-87 years, mean 71.7 years) and 12 late-stage OA patients (osteoarthritis; subjects: $60-84$ years, mean 64.7 years), classified according to American College of Rheumatology criteria.

\section{Isolation of Total RNA from Cartilage Tissue -}

cDNA Synthesis

Total RNA was isolated from cartilage tissue and transcribed into cDNA for real time PCR analysis, as described previously [11].
Gene Expression Profiling

Microarray analysis of RNA extracts was performed at the Münster University Genomics Center (Germany) using the GeneChip Human Genome U133 Plus 2.0 Array (Affymetrix, Santa Clara, Calif., USA) for more than 47,000 transcripts. Array experiments were performed according to protocols supplied by Affymetrix with $1 \mu \mathrm{g}$ of total RNA per sample. After PCR amplification of each sample, cDNA fragments were hybridized to a pre-equilibrated Affymetrix chip, washed, stained and scanned in the HP ChipScanner (Affymetrix). Primary normalization was performed with MAS5, as implemented in the Affymetrix data analysis software GCOS, according to the instruction of the manufacturer. MAD-scale normalization was applied for between-array normalization [12].

\section{Quantitative Real Time PCR}

mRNA expression levels were measured by real time quantitative PCR using an ABI Prism ${ }^{\circledR} 7700$ Sequence Detection System (Applied Biosystems, Foster City, Calif., USA). Primers (MWG Biotech, Ebersberg, Germany) and the TaqMan probe (Eurogentec, Seraing, Belgium) were designed using the Primer Express ${ }^{\circledR}$ software (Applied Biosystems). The sequences of primers and probes used are shown in table 1 . All experiments were performed in triplicates using titrated standard curves as described elsewhere [13]. Glyceraldehyde-3-phosphate dehydrogenase $(G A P D H)$ expression levels were used for normalization of gene expression values. Statistical evaluation of the expression levels was done by the nonparametric Wilcoxon-Mann-Whitney test. 
Fig. 1. Relative quantitation of $S O X$ transcript levels in normal and osteoarthritic cartilage. Total RNA was directly isolated from normal, early and late osteoarthritic cartilage, reverse transcribed and SOX transcript levels quantitated by real time PCR. Expression levels are shown as the ratio of $S O X$ transcripts to GAPDH transcripts. Data are presented as means \pm SE. ${ }^{* *} \mathrm{p}<0.01,{ }^{* * *} \mathrm{p}<0.001$.

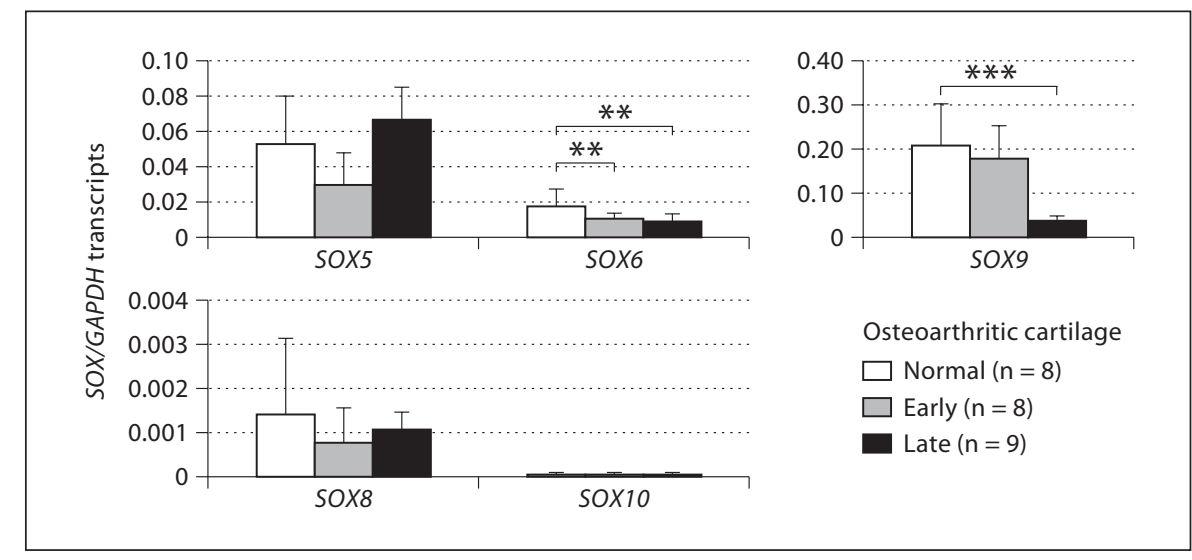

\section{Results}

\section{Gene Expression Profiling of SOX Transcription}

Factors in Normal and Osteoarthritic Cartilage of

\section{Adult Humans}

Gene expression profiling of adult human cartilage tissue, obtained from 13 normal and 12 osteoarthritic femoral condyles, using GeneChip technology showed no expression or a very weak expression for the majority of the analyzed SOX transcription factors (table 2).

The highest expression levels were observed for the SOX9 gene. Importantly, SOX9 expression was significantly reduced in the degenerated cartilage tissue taken from OA patients (fold change: 0.56 ). The SOX4 and SOX5 genes showed a moderate upregulation of transcript levels in osteoarthritic cartilage, starting from low basal expression levels in the normal tissue. SOX6 expression was below the detection limit, both in normal and degenerated tissue.

Regarding the genes closely related to SOX9, SOX8 showed a moderate expression in normal and osteoarthritic cartilage, while SOX10 expression was below the detection limit.

\section{Quantitation of SOX5, SOX6, SOX8, SOX9}

\section{and SOX10 Transcript Levels in Normal and}

\section{Osteoarthritic Articular Cartilage}

To verify and extend the data gained from the array experiments we quantified the expression levels for a selection of SOX transcription factors using a quantitative real time PCR approach (TaqMan). We chose the genes SOX5, SOX6 and SOX9, which were previously shown to play an important role in cartilage differentiation, and SOX8 and SOX10, which belong to the same SOX family subgroup as SOX9.
Table 2. Gene expression profiling of $S O X$ transcription factors in normal and osteoarthritic cartilage, with normalized signal intensities using arbitrary units

\begin{tabular}{lrlll}
\hline Gene & Gene ID & Normal & OA & $\begin{array}{l}\text { Fold change } \\
\text { OA/normal }\end{array}$ \\
& & & & \\
\hline SOX1 & 6656 & absent & absent & - \\
SOX2 & 6657 & absent & absent & - \\
SOX3 & 6658 & absent & absent & - \\
SOX4 & 6659 & 104 & 237 & 2.23 \\
SOX5 & 6660 & 152 & 332 & 2.17 \\
SOX6 & 55553 & absent & absent & - \\
SOX7 & 83595 & absent & absent & 0.73 \\
SOX8 & 30812 & 259 & 476 & 1.80 \\
SOX9 & 6662 & 2,935 & 1,582 & 0.56 \\
SOX10 & 6663 & absent & absent & - \\
SOX11 & 6664 & absent & 98 & - \\
SOX12 & 6666 & absent & absent & - \\
SOX13 & 9580 & absent & absent & - \\
SOX14 & 8403 & absent & absent & - \\
SOX15 & 6665 & absent & absent & - \\
SOX17 & 64321 & absent & absent & - \\
SOX18 & 54345 & absent & absent & - \\
SOX21 & 11166 & absent & absent & - \\
SOX30 & 11063 & absent & absent & - \\
\hline
\end{tabular}

Absent $=$ Below detection level.

Quantitative PCR data confirmed the comparatively high expression level of SOX9 in the normal cartilage tissue, amounting to approximately one fifth of GAPDH expression (fig. 1). Also, the data supported the substantial downregulation of $S O X 9$ expression in late osteoarthritic cartilage. Mildly degenerated cartilage (early OA) showed only a slight reduction in SOX9 expression levels. 
In comparison with SOX9 levels, SOX5 and SOX6 mRNA expression levels were notably lower. The low expression levels of SOX6 in normal cartilage were further reduced in early and late OA cartilage.

SOX8 and SOX10 transcript levels were very low in all tissues tested (less than $0.2 \%$ of GAPDH levels).

\section{Discussion}

Most of the 19 members of the SOX transcription factor family investigated in this study showed no or only a very weak expression in normal and osteoarthritic cartilage from human adults.

The strongest expression, amounting to approximately $20 \%$ of the GAPDH expression level, was found for SOX9, whose role in chondrocyte differentiation during development is well documented [for a review: 1]. The other two SOX proteins previously implicated in cartilage biology, SOX5 and SOX6, showed only moderate expression levels in adult cartilage. The SOX family members SOX8 and SOX10, which belong to the same subgroup as SOX9, were hardly detectable with highly sensitive techniques like quantitative real time PCR, which argues against an important role of these molecules in chondrocyte biology in adults.

Comparing the expression levels in normal and osteoarthritic cartilage the most interesting results are the substantial reduction of SOX9 transcript levels in OA, confirming previously reported data $[14,15]$, and the noticeable downregulation of SOX6 transcription.

Given the established role of SOX9 in chondrocyte differentiation during development, and the comparatively high expression in normal cartilage, an important role of SOX9 in the adult cartilage seems very likely. This is further supported by the fact that SOX9 expression gradually declines during the dedifferentiation of chondrocytes isolated from their surrounding matrix and cultured in vitro, a process reversible by the ectopic expression of SOX [16-18]. In this context, it is easily imaginable that the substantial reduction of SOX9 levels, which is evident in the osteoarthritic cartilage, might have an unfavorable effect on the phenotypic stability of the chondrocytes and thus ultimately on cartilage tissue integrity. Since SOX9 is known to be a potent inhibitor of the terminal hypertrophic differentiation, a reduced expression of SOX9 and its coactivator SOX6 might well be a decisive event in the hypertrophic differentiation of chondrocytes in the lowest zone of the osteoarthritic cartilage [19]. Although SOX5 expression showed a moder- ate upregulation in the diseased tissue, it is unlikely that this change could compensate for the loss of SOX6 and SOX9 expression given the low basic expression levels of SOX5. Likewise SOX4 expression was moderately elevated in OA, albeit starting from a very low basic expression in the normal tissue. Since no role has been described for SOX4 in cartilage biology, a biological importance for this upregulation seems improbable.

The reasons for the observed reduction of SOX 9 and SOX6 mRNA levels can only be speculated upon at the moment. It is known that SOX9 gene expression is influenced by many different signaling pathways. Proinflammatory cytokines like interleukin-1 alpha and tumor necrosis factor $\alpha$ potently inhibit SOX9 expression, while bone morphogenetic proteins (BMP-2), fibroblast growth factor (FGF), glucocorticoids and Hedgehog signaling positively stimulate SOX9 transcription [20-22]. Thus, deregulation of SOX9 expression in osteoarthritic cartilage might well be explained by an imbalance of these signaling pathways in the degenerating tissue. There is ample evidence for disturbances in several signaling pathways in OA ultimately favoring degradative events [for a review, see 23].

Alternatively, intrinsic control mechanisms like the epigenetic regulation of SOX promoter activity might be responsible for the observed downregulation of SOX9 and SOX6 transcript levels. Unfortunately, no data about $S O X$ promoter regulation by methylation is available for chondrocytes.

In summary, our data indicate that the role of SOX transcription factors in adult tissue is most probably restricted to a few members with SOX9 being the most prominent. Furthermore, the downregulation of SOX9 and SOX6 in the osteoarthritic cartilage suggests a role of these proteins in the phenotypic destabilization of osteoarthritic chondrocytes.

\section{Acknowledgements}

We are grateful to Brigitte Bau and Anke Nehlen for excellent technical assistance. This work was supported by the German National Science Foundation (grant AI20/7-1). 


\section{References}

1 Ikeda T, Kawaguchi H, Kamekura S, Ogata N, Mori Y, Nakamura K, Ikegawa S, Chung UI: Distinct roles of Sox5, Sox6, and Sox9 in different stages of chondrogenic differentiation. J Bone Miner Metab 2005;23:337-340.

${ }_{2}$ Bi W, Huang W, Whitworth DJ, Deng JM, Zhang Z, Behringer RR, de Crombrugghe B: Haploinsufficiency of Sox9 results in defective cartilage primordia and premature skeletal mineralization. Proc Natl Acad Sci USA 2001;98:6698-6703.

3 Akiyama H, Chaboissier MC, Martin JF, Schedl A, de Crombrugghe B: The transcription factor Sox 9 has essential roles in successive steps of the chondrocyte differentiation pathway and is required for expression of Sox5 and Sox6. Genes Dev 2002;16:28132828.

-4 Foster JW, Dominguez-Steglich MA, Guioli S, Kwok C, Weller PA, Stevanovic M, Weissenbach J, Mansour JD, Young ID, Goodfellow PN, Brook JD, Schafer AJ: Campomelic dysplasia and autosomal sex reversal caused by mutations in an SRY-related gene. Nature 1994;372:525-530.

5 Ng L-J, Wheatley S, Muscat GEO, ConwayCampbell J, Bowles J, Wright E, Bell DM, Tam PPL, Cheah KSE, Koopman P: SOX9 binds DNA, activates transcription, and coexpresses with type II collagen during chondrogenesis in the mouse. Dev Biol 1997;183: 108-121.

-6 Zhao Q, Eberspaecher H, Lefebvre V, de Crombrugghe B: Parallel expression of Sox 9 and Col2a1 in cells undergoing chondrogenesis. Dev Dyn 1997;209:377-386.

7 Bell DM, Leung KKH, Wheatley SC, Ng L-J, Zhou S, Wing-Ling K, Sham MH, Koopman P, Tam PPL, Cheah KSE: SOX9 directly regulates the type-II collagen gene. Nat Genet 1997; 16:174-178.
-8 Lefebvre V, Huang W, Harley VR, Goodfellow PN, de Crombrugghe $\mathrm{B}$ : SOX9 is a potent activator of the chondrocyte-specific enhancer of the Proo1(II) collagen gene. Mol Cell Biol 1997;17:2336-2346.

-9 Lefebvre V, Li P, de Crombrugghe B: A new long form of Sox 5 (L-Sox-5), Sox6 and Sox9 are coexpressed in chondrogenesis and cooperatively activate the type II collagen gene. EMBO J 1998;17:5718-5733.

10 Aigner T, Zien A, Gehrsitz A, Gebhard PM, McKenna LA: Anabolic and catabolic gene expression pattern analysis in normal versus osteoarthritic cartilage using complementary DNA-array technology. Arthritis Rheum 2001;44:2777-2789.

11 McKenna LA, Gehrsitz A, Soeder S, Eger W, Kirchner T, Aigner T: Effective isolation of high-quality total RNA from human adult articular cartilage. Anal Biochem 2000;286: 80-85.

12 Dudoit S, Yang YH: Bioconductor R packages for exploratory analysis and normalization of cDNA microarray data; in Parmigiani G, Garett ES, Irizarry RA, Zeger SL (eds): The Analysis of Gene Expression Data. New York, Springer, 2003, pp 73-101.

13 Bau B, Gebhard PM, Haag J, Knorr T, Bartnik E, Aigner T: Relative messenger RNA expression profiling of collagenases and aggrecanases in human articular chondrocytes in vivo and in vitro. Arthritis Rheum 2002;46: 2648-2657.

14 Aigner T, Gebhard PM, Schmid E, Bau B, Harley V, Pöschl E: SOX9 expression does not correlate with type II collagen expression in adult articular chondrocytes. Matrix Biol 2003;22:363-372.

15 Tchetina EV, Squires G, Poole AR: Increased type II collagen degradation and very early focal cartilage degeneration is associated with upregulation of chondrocyte differentiation related genes in early human articular cartilage lesions. J Rheumatol 2005;32: 876-886.
16 Stokes DG, Liu G, Dharmavaram R, Hawkins D, Piera-Velazquez S, Jimenez SA: Regulation of type-II collagen gene expression during human chondrocyte de-differentiation and recovery of chondrocyte-specific phenotype in culture involves Sry-type highmobility-group box (SOX) transcription factors. Biochem J 2001;360:461-470.

17 Tew SR, Li Y, Pothacharoen P, Tweats LM, Hawkins RE, Hardingham TE: Retroviral transduction with SOX9 enhances re-expression of the chondrocyte phenotype in passaged osteoarthritic human articular chondrocytes. Osteoarthritis Cartilage 2005; 13:80-89.

18 Hardingham T, Tew S, Murdoch A: Tissue engineering: chondrocytes and cartilage. Arthritis Res 2002;4(suppl 3):S63-S68.

19 Girkontaité I, Frischholz S, Lammi P, Wagner K, Swoboda B, Aigner T, von der Mark K: Immunolocalization of type $\mathrm{X}$ collagen in normal fetal and adult osteoarthritic cartilage with monoclonal antibodies. Matrix Biol 1996;15:231-238.

20 Murakami S, Lefebvre V, de Crombrugghe B: Potent inhibition of the master chondrogenic factor Sox 9 gene by interleukin-1 and tumor necrosis factor- $\alpha$. J Biol Chem 2000;275: 3687-3692.

21 Uusitalo H, Hiltunen A, Ahonen M, Gao TJ, Lefebvre V, Harley V, Kahari VM, Vuorio E: Accelerated up-regulation of L-Sox5, Sox6, and Sox 9 by BMP-2 gene transfer during murine fracture healing. J Bone Miner Res 2001;16:1837-1845.

22 Sekiya I, Koopman P, Tsuji K, Mertin S, Harley V, Yamada Y, Shinomiya K, Nifuji A, Noda M: Dexamethasone enhances SOX9 expression in chondrocytes. J Endocrinol 2001;169:573-579.

23 Sandell LJ, Aigner T: Articular cartilage and changes in arthritis. An introduction: cell biology of osteoarthritis. Arthritis Res 2001;3: 107-113. 